\title{
An Analysis of Dispersion and Dissipation Properties of Hermite Methods and its Application to Direct Numerical Simulation of Jet Noise
}

\author{
Chang Young Jang * Daniel Appelö ${ }^{\dagger} \quad$ Tim Colonius ${ }^{\ddagger} \quad$ Thomas Hagstrom ${ }^{\S}$ \\ Matthew Inkman
}

\begin{abstract}
The dissipative and dispersive properties of Hermite methods are analyzed by a modified equation approach and by direct computation of the dispersion relations for the discrete modes of the scheme. The two approaches lead to the same results for well-resolved modes but are quantitatively different at the finest scales. The resolution requirements, obtained from the analysis, for Hermite schemes are compared to those of typical high-resolution difference formulas. The results from the analysis are also used to predict the resolution requirements for a simulation at $\operatorname{Re} \sim 3600$. The validity of the prediction is confirmed by numerical experiments.
\end{abstract}

\section{Introduction}

Ever increasing computer speeds makes Direct Numerical Simulation (DNS) of turbulent flows, including turbulent jet noise, at increasingly higher Reynolds numbers (Re) possible. However, to reach the high Reynolds number encountered in engineering applications (be it by DNS or LES), algorithms that scale to the Petascale, and eventually Exascale, must be developed. If the end goal is to perform DNS/LES of turbulent flows, such algorithms must also have high resolution and minimal dispersion and dissipation in order to resolve the dynamically relevant scales of motion and to accurately represent the radiated acoustic field.

This paper will report on recent progress on the development, analysis and application of a class of methods known as Hermite methods ${ }^{8}$ which are arbitrarily high order accurate, and very suitable for parallel architectures. This work is a continuation of our research program ${ }^{3-5,9}$ on Hermite methods and their application to jet noise.

We analyze the accuracy of Hermite methods as characterized by their dissipative and dispersive properties. Two complementary techniques will be employed. The first one is based on a modified equation approach while the second directly computes the dispersion relations for the discrete modes of the scheme. For well-resolved modes these two analyses lead to the same results. However, at the finest scales they are quantitatively different. We also use the calculated dispersion relation to compare the resolution requirements for Hermite schemes to those of typical high-resolution difference formulas. The results from the analysis are used to predict the resolution requirements for a simulation at $\operatorname{Re} \sim 3600$, which is then confirmed by numerical experiments.

\section{Dissipation and Dispersion}

To verify that direct numerical simulations are well-resolved and help predict resolution requirements for future runs it is useful to study the dissipation and dispersion characteristics of the Hermite methods. Here we will focus on the approximation of the advective terms in the equation, recognizing that a complete theory

\footnotetext{
*Graduate Student, Department of Mathematics, Southern Methodist University.

$\dagger$ Assistant Professor, Department of Mathematics and Statistics, University of New Mexico.

$\ddagger$ Professor, Department of Mechanical Engineering, California Institute of Technology, AIAA Senior Member.

$\S$ Professor, Department of Mathematics, Southern Methodist University, AIAA Senior Member.

ฯGraduate Student, Department of Mechanical Engineering, California Institute of Technology.
} 
must also include the errors in the approximation of the terms involving viscosity and heat conduction. Thus we consider the model problem

$$
\frac{\partial u}{\partial t}+\frac{\partial u}{\partial x}=0
$$

We recall the basic structure of Hermite methods applied to (1). At time $t_{n}$ we suppose that at nodes, $x_{j}$, the coefficients $U_{j}^{k}\left(t_{n}\right)$,

$$
U_{j}^{k}\left(t_{n}\right) \approx \frac{h^{k}}{k !} \frac{\partial^{k} u}{\partial x^{k}}\left(x_{j}, t_{n}\right), \quad j=0, \ldots, m,
$$

are known. (Here $h=x_{j+1}-x_{j}$ is the grid spacing.) The data is evolved over a halfstep, $t_{n} \rightarrow t_{n+1 / 2}=$ $t_{n}+\gamma \frac{h}{2}$, to produce the approximate solution on the staggered grid, $x_{j+1 / 2}=x_{j}+\frac{h}{2}$. (Here $\gamma=\frac{\Delta t}{h}$ is the CFL number.) The recipe for evolution is as follows:

i. Compute the Hermite (sometimes called Birkhoff-Hermite) interpolant of the vertex polynomials. The interpolant is a tensor-product polynomial of degree $2 m+1$ in each variable. Globally we have a $C^{m}$ piecewise polynomial, $S_{n}(x)$, approximating $u\left(x, t_{n}\right)$.

ii. Solve (1) with the local polynomial data to produce the data

$$
U_{j+1 / 2}^{k}\left(t_{n+1 / 2}\right) \approx \frac{h^{k}}{k !} \frac{\partial^{k} u}{\partial x^{k}}\left(x_{j+1 / 2}, t_{n+1 / 2}\right), \quad j=0, \ldots, m .
$$

Here the local evolution step can be carried out exactly using temporal Taylor series. For our NavierStokes calculations it is more efficient to use multiple substeps of a standard Runge-Kutta method.

This process is repeated on the dual grid to produce the approximate solution on the primary grid at time $t_{n+1}$.

The most attractive feature of Hermite schemes is the possibility to evolve large chunks of data independently over relatively large time steps. This feature enables implementations to run with large computationto-communication ratios. In addition, we have found that the stability properties of Hermite methods are excellent, both in terms of avoiding nonlinear instabilities and advecting nonsmooth flow features with little oscillation but also without excessive artificial diffusion. See Appelö and Hagstrom ${ }^{4,9}$ for examples.

The basic properties of Hermite methods are established in Goodrich et al. ${ }^{8}$ The most important are:

i. The method is stable with exact (or sufficiently accurate) local time stepping so long as waves cannot propagate from the cell boundaries to the cell center in a half time step. Note that this result is independent of the degree, $m$.

ii. The method is dissipative in the sense that, for the Cauchy problem or with periodic boundary conditions, the piecewise polynomial interpolants, $S\left(x, t_{n}\right)$, satisfy

$$
\left\|\frac{\partial^{m+1} S\left(\cdot, t_{n+1}\right)}{\partial x^{m+1}}\right\|_{L^{2}}^{2} \leq\left\|\frac{\partial^{m+1} S\left(\cdot, t_{n+1 / 2}\right)}{\partial x^{m+1}}\right\|_{L^{2}}^{2} \leq\left\|\frac{\partial^{m+1} S\left(\cdot, t_{n}\right)}{\partial x^{m+1}}\right\|_{L^{2}}^{2} .
$$

iii. For smooth solutions the error satisfies

$$
\left\|u\left(\cdot, t_{n}\right)-S\left(\cdot, t_{n}\right)\right\|_{L^{2}} \leq C h^{2 m+1}\|u(\cdot, 0)\|_{H^{2 m+2}},
$$

for some universal constant $C$.

Our detailed analysis of discretization error for Hermite methods applied to (1) involves the behavior of the discrete dispersion relation with wavenumber. This is somewhat more involved than analogous studies for difference approximations, as discussed in the context of aeroacoustics by Colonius and Lele. ${ }^{6}$ Firstly, we cannot semidiscretize in time. Thus the dissipation/dispersion error rates are calculated based on the errors produced in a single half-step. Second, again in contrast with standard difference methods, the eigenmodes of the evolution operator do not directly correspond to basic Fourier modes, $u=e^{i k x}$. Thus the error in propagating $e^{i k x}$ involves both the difference between the Fourier mode and the related numerical mode and the difference in their propagation characteristics. (Such issues naturally arise in the consideration of spectral element methods; see for example the work of Ainsworth. ${ }^{1,2}$ ) 
Here we will study the discretization error using two complementary techniques. For the first we will employ a modified equation derived by Appelö and Hagstrom. ${ }^{4}$ This yields an approximate analytic formula for error propagation based on average Hermite interpolation error. As such it predicts a purely dissipative error and mixes in the effects of the modal errors and their dissipation/dispersion relations. For the second we directly compute the dipersion relations for the discrete modes themselves. For well-resolved modes these two analyses lead to the same results. However, at the finest scales they are quantitatively different.

As we are particularly concerned with the application of these results to the DNS of jet noise, we will present data for $m=3$ and $m=4$. Freund [7, Figure 5] displays energy spectra indicating that for wave numbers $k R \sim 70$ the energy is negligible. (Here $R$ is the jet radius.) Our results below show that for $m=3$ and our current grid the numerical dissipation becomes comparable to the physical dissipation only at these fine scales and for $m=4$ physical dissipation is dominant. Moreover, the dispersion error is an order of magnitude smaller than the dissipation error. Thus the conclusion based on these considerations is that the flow is well-resolved.

We also make some comparison between the resolution of Hermite schemes and the various difference methods discussed by Colonius and Lele. ${ }^{6}$ We note that we expect that a well-implemented Hermite method will be highly efficient in terms of communication time per degree-of-freedom. Thus we need only verify that the resolution requirements are not degraded relative to competitive methods.

\section{Modified Equation}

When a PDE is approximated by a numerical method it is often useful to find the modified equation to understand the qualitative behavior of the numerical solution. The modified equation is a PDE related to the original PDE but with additional term(s) that accounts for the leading order error(s) introduced when the numerical method is used to solve the original PDE. A detailed discussion of the theory and use of modified equations can be found in Hedstrom. ${ }^{10}$

The analysis in ref. 4 derives such an equation for Hermite methods applied to (1) by looking at the average error over a computational cell. It can be shown that this error is due solely to repeated Hermite interpolation, which dissipates the $L^{2}$-norm of the $m+1$ spatial derivative. Based on the leading order error caused by that process it is thus argued that an appropriate modified equation is

$$
\frac{\partial v}{\partial t}+\frac{\partial v}{\partial x}=(-1)^{m} \nu_{h} \frac{\partial^{2 m+2} v}{\partial x^{2 m+2}}
$$

where

$$
\nu_{h}=\frac{1}{\gamma} \frac{h^{2 m+1}}{4^{m+1}(2 m+2) !} \frac{\sqrt{\pi} \Gamma(m+2)}{\Gamma(m+5 / 2)},
$$

with $h$ the cell width and $\gamma=\frac{\Delta t}{h}$ the CFL number.

Note that (6) only predicts the leading order dissipation error. Thus we can only use it to compare the numerical dissipation for Fourier modes to the physical dissipation due to viscosity (here at the reference Reynolds number, $R e_{D}=3600$.) The results for

$$
h=.072 R, \quad \gamma=.25
$$

are presented in Figure 1. Except at the finest scales the numerical dissipation as predicted by (6) is negligible compared with the physical dissipation.

\section{Modal Analysis}

To carry out a discrete modal analysis we consider a periodic problem. The evolution of the discrete data over a half time step is equivalent to the multiplication by the block circulant matrix $A(\gamma, m)$ :

$$
A=\left(\begin{array}{ccccc}
S_{L} & S_{R} & 0 & \cdots & 0 \\
0 & S_{L} & S_{R} & 0 & \vdots \\
\vdots & \vdots & \ddots & \ddots & \vdots \\
\vdots & \vdots & \vdots & \ddots & \vdots \\
S_{R} & 0 & \cdots & \cdots & S_{L}
\end{array}\right)
$$




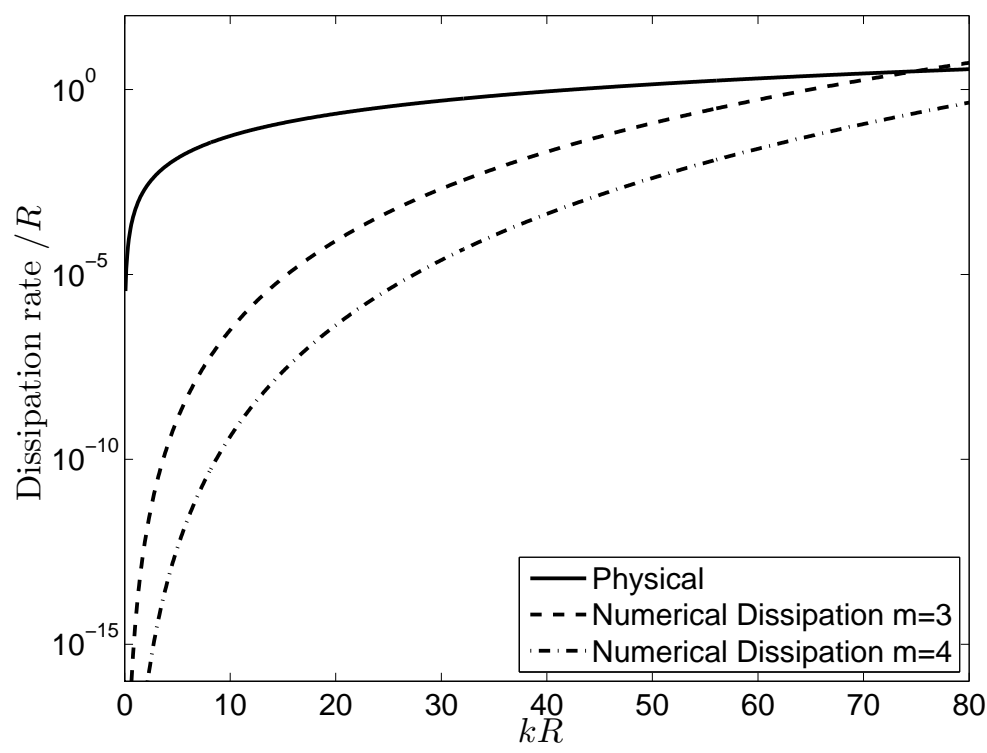

Figure 1. Comparison of viscous dissipation versus numerical dissipation as predicted by (6).

where $S_{L, R} \in \mathbb{R}^{(m+1) \times(m+1)}$ are given by

$$
\left(S_{L} \quad S_{R}\right)=T \cdot \exp \left(-\frac{d}{d x} \cdot \frac{\Delta t}{2}\right) \cdot H
$$

where $H \in \mathbb{R}^{(2 m+2) \times(2 m+2)}$ is the Hermite interpolation matrix and $T$ is the truncation of a degree- $(2 m+1)$ Taylor polynomial to degree $m$. Note that we have assumed locally exact time-stepping, which for (1) is achieved by using a temporal Taylor series of degree $2 m+1$.

Using the discrete Fourier series we can write data at each node

$$
w_{j}=\sum_{k=-N / 2}^{N / 2} \hat{w}_{k} e^{2 \pi i k j / N},
$$

where

$$
w_{j}=\left[\begin{array}{c}
u\left(x_{j}\right) \\
\partial_{x} u\left(x_{j}\right) \\
\partial_{x}^{2} u\left(x_{j}\right) \\
\vdots \\
\partial_{x}^{l} u\left(x_{j}\right)
\end{array}\right], \quad l=0, \ldots, m .
$$

As each mode will correspond to a single value of $k$ (though there will be $m+1$ distinct modes for each $k$, approximating physical modes at the higher, aliased wavenumbers), the evolution of the discrete data over a half time step in terms of the Fourier modes takes the form (using the expression of the discrete Fourier series in (11))

$$
e^{2 \pi i k j / N} S_{L} \hat{w}_{k}+e^{2 \pi i k(j+1) / N} S_{R} \hat{w}_{k}=e^{\pi i k / N+2 \pi i k j / N} e^{\alpha} \hat{w}_{k} .
$$

Simplifying further we deduce the modal eigenvalue problem

$$
\hat{H}(k, c, m) \hat{w}_{k}=e^{\alpha} \hat{w}_{k},
$$

where

$$
\begin{aligned}
& \hat{H}(c, m)=e^{-i \theta / 2} S_{L}+e^{i \theta / 2} S_{R}, \quad \hat{H} \in \mathbb{R}^{(m+1) \times(m+1)} \\
& \theta=2 \pi k / N \in[0(m+1) \pi] .
\end{aligned}
$$


The eigenvalue, $e^{\alpha}$, is an approximation to the exact solution operator

$$
e^{-i \gamma \theta / 2}
$$

Thus the modal dissipation rate and dispersion error are given by:

$$
\text { Dissipation Rate }=\frac{2}{\gamma} \Re \alpha, \text { Dispersion Error }=\theta+\frac{2}{\gamma} \Im \alpha .
$$

These errors are plotted in Figure 2 and Figure 3. We see that the dissipation rates at the fine scale are about an order of magnitude smaller than those predicted by (6) and the dispersion errors are even smaller. Thus again the conclusion is that our current grid is more than sufficient to resolve the flow.

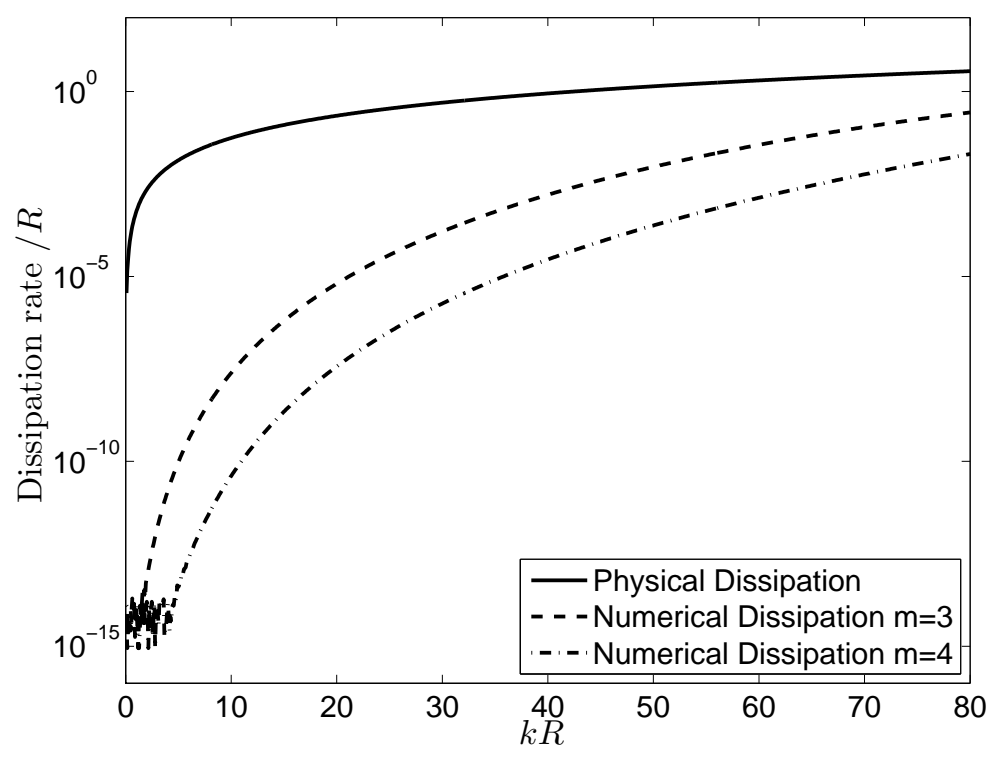

Figure 2. Comparison of viscous dissipation versus numerical dissipation as predicted by (18).

\section{Comparisons with Other Methods}

In order to compare the resolution of Hermite methods with other spatial discretization schemes as well as to better understand their performance as $m$ and $\gamma$ varies, we plot in Figure 4 and Figure 5 the number of degrees of freedom per wavelength versus dissipation error. In Figure 4 we fix $\gamma$ and vary $m$. We see that at an error level of $10^{-3}$ this number varies from roughly 6 through 9 as $m$ varies from 3 through 7 . At an error rate of $10^{-4}$ the range is roughly 7 through $11-12$.

In Figure 5 we fix $m=3$, the lowest order method used in the simulations, and vary $\gamma$ between 0.1 and 0.9. As expected, accuracy increases with increasing time step. So long as $\gamma<1$, the only errors are due to reinterpolation. As the time step is increased, the interpolation rate is decreased. Precisely, for an error level of $10^{-3}$ the number of degrees-of-freedom nedded varies from $7-8$ to $11-12$. For an error level of $10^{-4}$ it varies from roughly 10 to 14 . Thus varying $\gamma$ has as strong an effect as varying $m$ over a wide range. Note that the resolution estimates above were made for $\gamma=.25$, which is somewhat smaller than what is used in the simulations presented below.

In Figure 6 and Figure 7 we superpose these graphs with the dispersion error graphs for standard central and compact difference schemes as well as the DRP scheme of Tam and Webb. ${ }^{12}$ Note that we have not included the effects of time stepping for the difference schemes, so CFL-dependence is absent.

Figure 6 , computed with $\gamma=0.8$ with the Hermite methods, demonstrates that the resolution of the Hermite methods compares favorably with most of the alternative discretizations considered. At error levels of $10^{-3}$ and $10^{-4}$ only the sixth order compact sheme has competitive accuracy - roughly comparable to Hermite with $m=6$ for the coarser tolerance and $m=4$ at the finer tolerance. At finer tolerances the higher order Hermite methods are more accurate. 


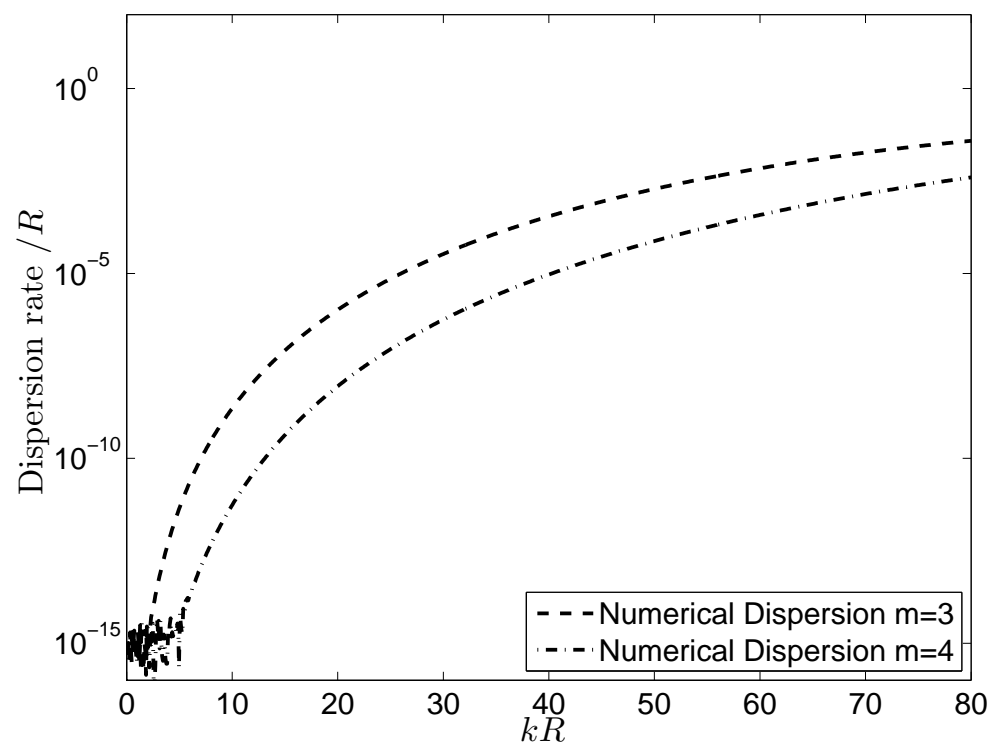

Figure 3. Dispersion dissipation for $m=3,4$ as predicted by (3).

In Figure 7 we compare the lowest order Hermite method we have used, namely the 7 th order scheme, and various $\gamma$ to the finite difference formulas. Again we note that the effects of temporal discretization are not factored into the central difference graphs. Again we see that the resolution of the Hermite scheme is better than the central difference formulas at the fine tolerances and for all values of $\gamma$ with the exception of the 6th order compact method. At the coarser tolerance of $10^{-3}$ the 6th order explicit method is also comparable.

We note, however, that the Hermite schemes have the advantage of minimizing communication costs, while the compact schemes, in their basic form, require global communication. Thus we are confident that more efficient prallel implementations of the Hermite method can be realized. Even for serial computations, we have shown that Hermite methods are more efficient than difference methods even in simple geometry for all but the least stringent error requirements. See, in particular, Figure 3 in Appelö et al. ${ }^{5}$

\section{Application to DNS of Jet Noise}

To test the above analysis when applied to a simulation of the full compressible Navier-Stokes equations we perform a refinement study of a jet similar to Freund's with $R e=\rho_{\mathrm{j}} U_{\mathrm{j}} / \mu_{\mathrm{j}}=3600$ at Mach 0.86 . The equations are non-dimensionalized by jet diameter, $D_{\mathrm{j}}$, density and sound speed in the quiescent flow, $\rho_{\infty}, a_{\infty}^{2}$ and by jet exit velocity $U_{j}$. The equations are solved in a computational domain consisting of a box $\left(x / D_{\mathrm{j}}, y / D_{\mathrm{j}}, z / D_{\mathrm{j}}\right) \in[-1,19] \times[-5.5,5.5]^{2}$. Towards the end of the domain we add a damping layer to absorb outgoing disturbances. The layer is terminated using characteristic boundary conditions. The nozzle is modeled by the same momentum forcing as Freund. ${ }^{7}$

For the non-refined case we discretize the equations we use a seventh order accurate method in space and the classic fourth order accurate Runge-Kutta method in time. The computational domain is discretized on a stretched tensor grid consisting of $161 \times 126 \times 126$ points. The grid has a minimal grid spacing roughly four times larger than Freund. The refinement consists of increasing the approximation order in space from $7(m=3)$ to $9(m=4)$ and decreasing the time step by a factor of 0.3 .

The refined computation is relatively expensive so we start the comparison 720 time steps into the (coarse) simulation. At this time the initial data, based on mean flow profiles from Stromberg, ${ }^{11}$ is in the first stages of transitioning into a turbulent flow. As the initial data does not satisfy the equations there is a significant rearrangement of the flow which generates generates a plug of high speed fluid around 7 diameters downstream the nozzle. When this plug breaks down it generates fine scale acoustic waves that are challenging to resolve. We note that once such transients have washed out the flow will be easier to 


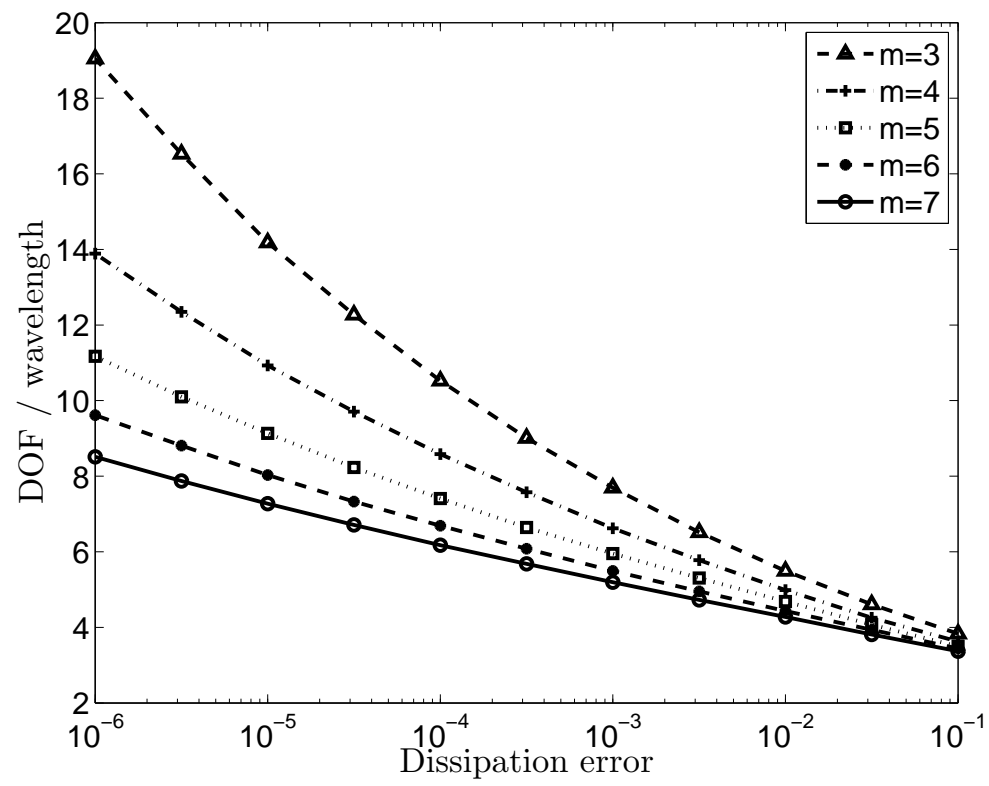

Figure 4. The number of degrees of freedom per wavelength versus dissipation error using Hermite methods applied to the model equation with $\gamma=0.8$ and $m$ ranging from 3 to 7 .

resolve.

As a first test we compare the magnitude of the velocity along the line $y=0, z=0.44 D_{\mathrm{j}}$ for $0 \leq x \leq 10 D_{\mathrm{j}}$ at 150 time steps (on the fine grid) after the start of the comparison. The results, displayed in Figure 8, show that the coarse simulation with $m=3$ well resolves the hydrodynamic part of the test flow.

For the second, more challenging, test we compare the pressure perturbation along the lines between the points $\left(0,0, D_{\mathrm{j}}\right),\left(10 D_{\mathrm{j}}, 0,3 D_{\mathrm{j}}\right)$ and $\left(0,0,4 D_{\mathrm{j}}\right),\left(10 D_{\mathrm{j}}, 0,4 D_{\mathrm{j}}\right)$. The results can be found in Figure 9 . As can be seen in the Figure the agreement is good but there is a distinguishable difference. This indicates that the resolution is just enough to represent the pressure perturbations.

\section{Summary}

We have presented an analysis of the dissipative and dispersive properties and their effect on the accuracy of Hermite methods. The analysis was performed using two different and complementary techniques. The first one, based on a modified equation approach, accurately predicts the dissipative behavior of the method for well resolved modes but over predicts the numerical dissipation for poorly resolved modes. The second method directly computes the dispersion relations for the discrete modes of the scheme. The results using the second method was used to compare the resolution requirements for Hermite schemes to those of typical high-resolution difference formulas. Finally we used our analysis to predict the resolution requirements for a simulation at $\operatorname{Re} \sim 3600$. The numerical experiments confirm the validity of the analysis.

This material is based upon work supported, in part, by the National Science Foundation under Grants OCI-0905045 and OCI-0904773 and, in part, by ARO grant W911NF-09-1-0344. Any opinions, findings, and conclusions or recommendations expressed in this material are those of the authors and do not necessarily reflect the views of the National Science Foundation

\section{References}

${ }^{1}$ M. Ainsworth. Discrete dispersion relation for hp-version finite element approximation at high wave number. SIAM $J$. Num. Anal., 42:553-575, 2004.

${ }^{2}$ M. Ainsworth. Dispersive and dissipative behavior of high-order discontinuous Galerkin finite element methods. J. Comput. Phys., 198:106-130, 2004.

${ }^{3}$ D. Appelö, T. Colonius, T. Hagstrom, and M. Inkman. Development of arbitrary-order hermite methods for simulation 


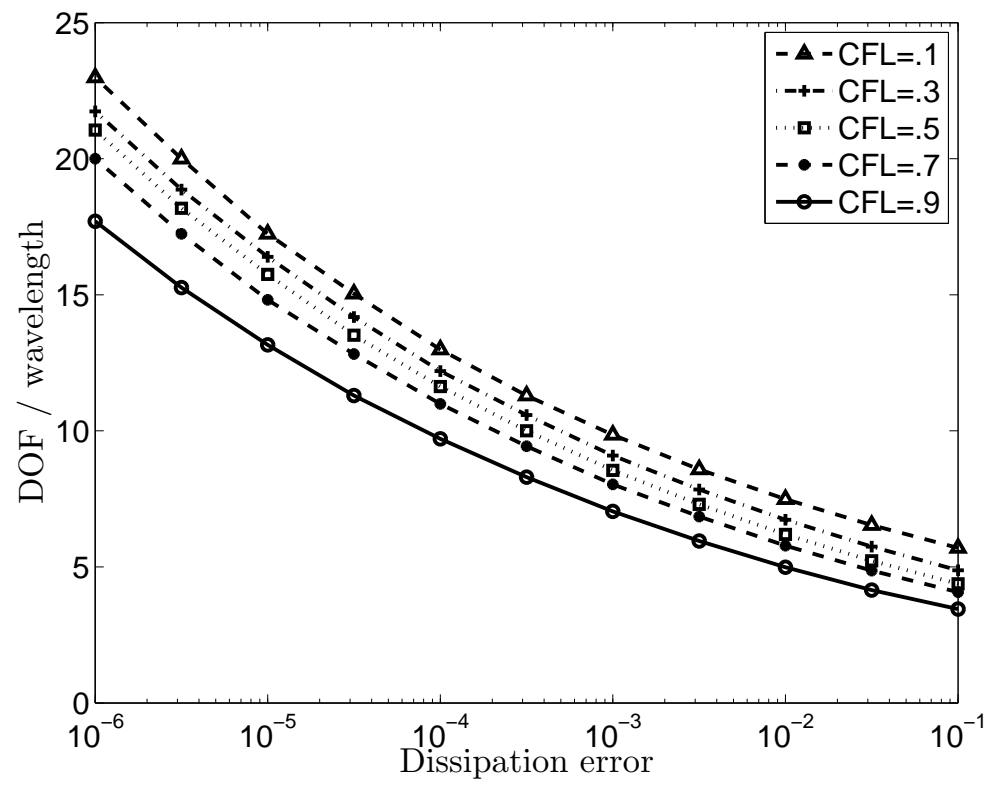

Figure 5. The number of degrees of freedom per wavelength versus dissipation error using Hermite methods applied to the model equation with the fixed $m=3$ and $\gamma$ varying from 0.1 to 0.9 .

and analysis of turbulent jet noise. Procedia Engineering, IUTAM Symposium on Computational Aero-Acoustics for Aircraft Noise Prediction, University of Southampton, 29-31 March, 2010.

${ }^{4}$ D. Appelö and T. Hagstrom. On advection by Hermite methods. Paciffic Journal Of Applied Mathematics, 4(2), 2011.

${ }^{5}$ D. Appelö, M. Inkman, T. Hagstrom, and T. Colonius. Hermite methods for aeroacoustics: Recent progress. In 17th AIAA/CEAS Aeroacoustics Conference (32nd AIAA Aeroacoustics Conference), Portland, Oregon, June 5-8, 2011, number AIAA-2011-2757, 2011.

${ }^{6}$ T. Colonius and S. Lele. Computational aeroacoustics: progress on nonlinear problems of sound generation. Progress in Aerospace Sciences, 40:345-416, 2004.

${ }^{7}$ J. Freund. Noise sources in a low Reynolds number jet at Mach 0.9. J. Fluid Mech., 438:277-305, 2001.

${ }^{8} \mathrm{~J}$. Goodrich, T. Hagstrom, and J. Lorenz and. Hermite methods for hyperbolic initial-boundary value problems. Math. Comp., 75(254):595-630, 2005.

${ }^{9} \mathrm{~T}$. Hagstrom and D. Appelö. Experiments with hermite methods for simulating compressible flows: Runge-kutta timestepping and absorbing layers. In 13th AIAA/CEAS Aeroacoustics Conference, number 2007-3505, 2007.

${ }^{10} \mathrm{G}$. W. Hedstrom. Models of difference schemes for $u_{t}+u_{x}=0$ by partial-differential equations. Mathematics of Computation, 29:969-977, 1975.

${ }^{11}$ J.L. Stromberg, D.K. McLaughlin, and T.R. Troutt. Flow field and acoustic properties of a Mach number 0.9 jet at a low Reynolds number. J. of Sound and Vib., 72:159-176, 1980.

${ }^{12} \mathrm{C} . \mathrm{K} . \mathrm{W}$. Tam and J. C. Webb. Dispersion-relation-preserving finite-difference schemes for computational acoustics. Journal of Computational Physics, 107(2):262-281, August 1993. 


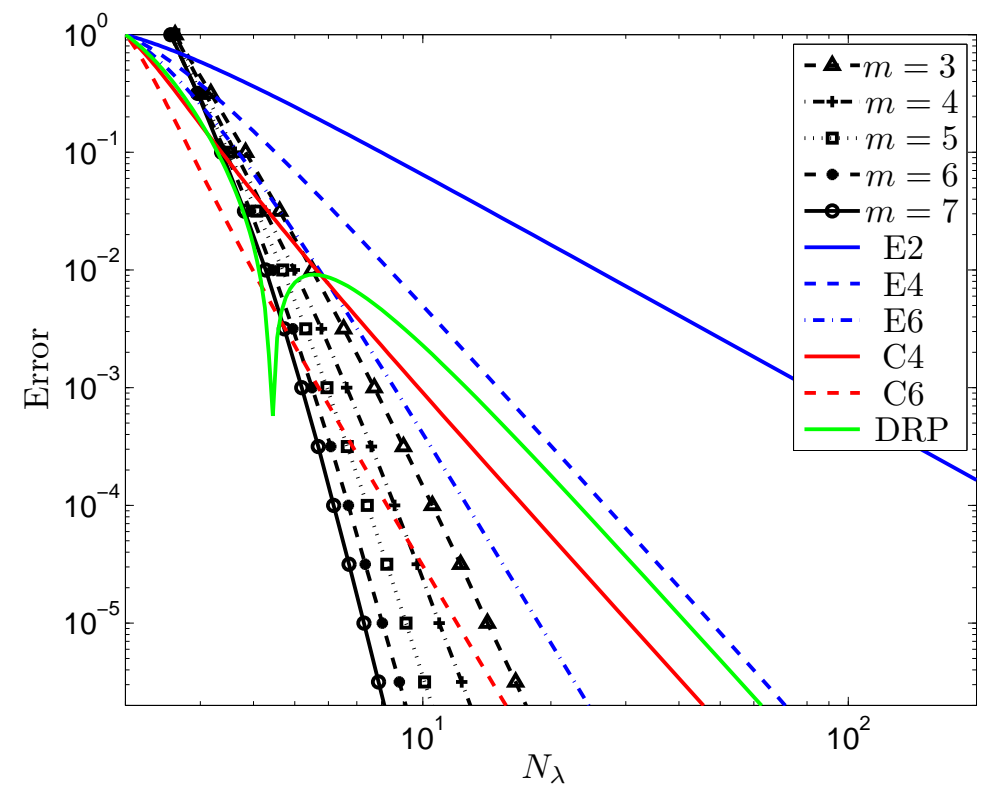

Figure 6. The number of degrees of freedom per wavelength versus dissipation error using Hermite methods applied to the model equation with $\gamma=0.8$ and $m$ ranging from 3 to 7 . Here we compare with explicit central difference methods, compact difference methods, and the DRP scheme.

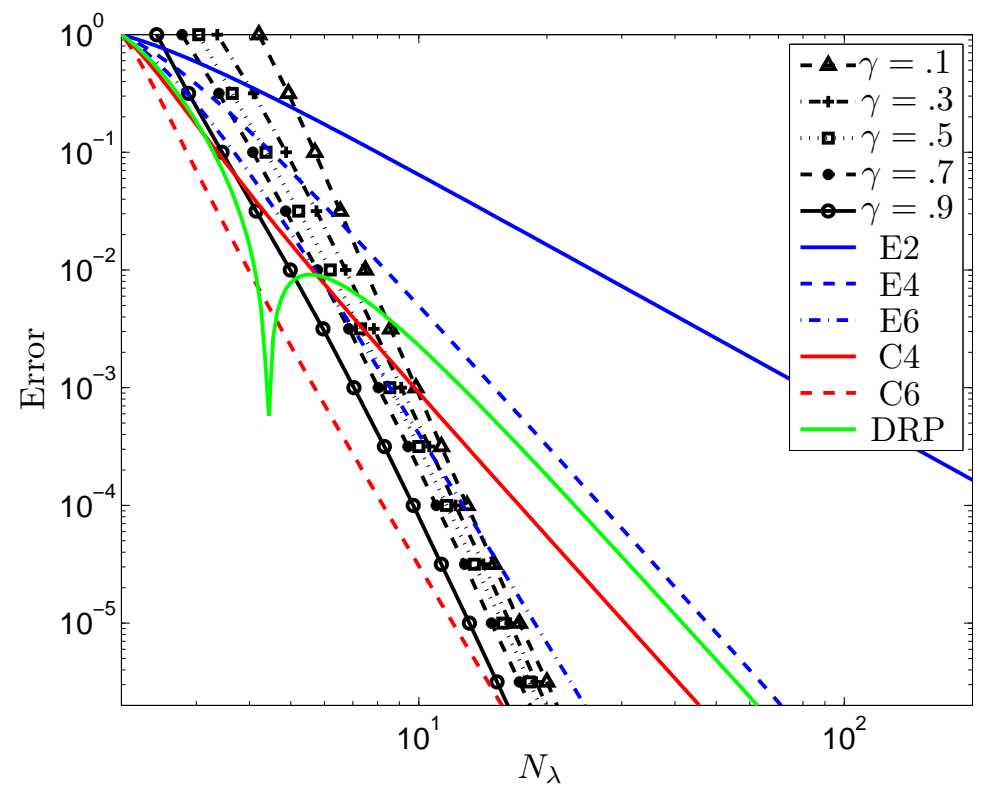

Figure 7. The number of degrees of freedom per wavelength versus dissipation error using Hermite methods applied to the model equation with the fixed $m=3$ and $\gamma$ varying from 0.1 to 0.9 . Here we compare with explicit central difference methods, compact difference methods, and the DRP scheme. 


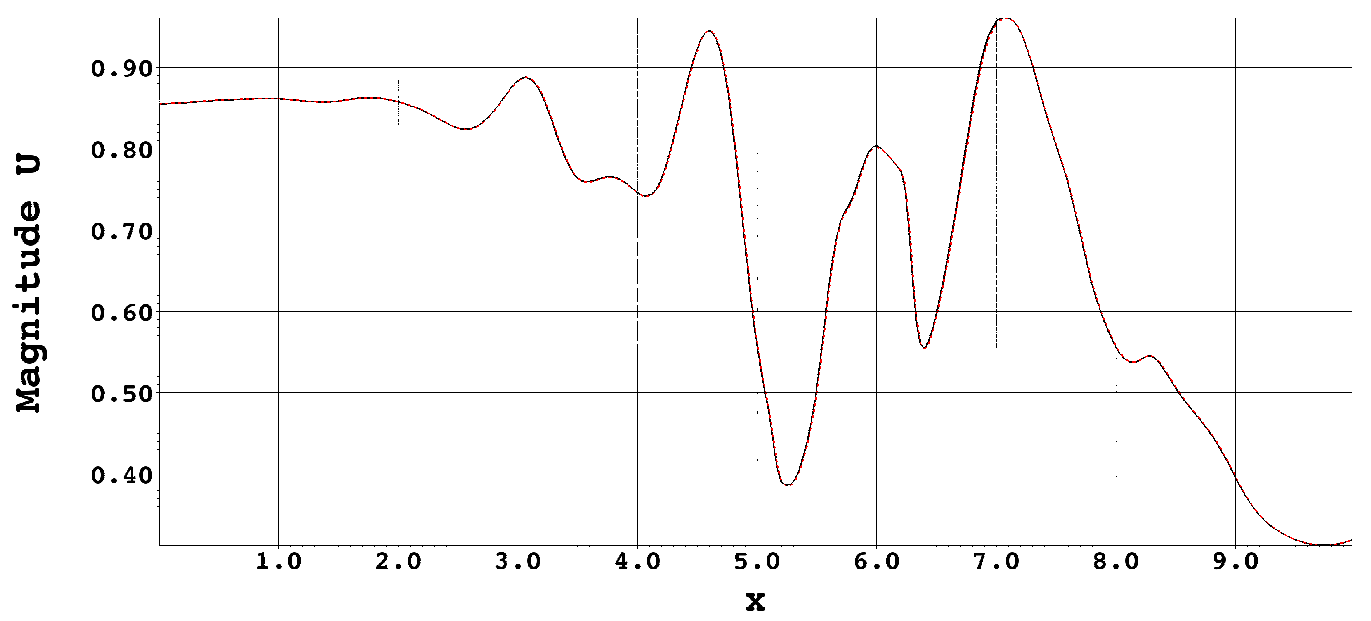

Figure 8. Line plot of the magnitude of the velocity along the line $y=0.44 D_{\mathrm{j}}$ for $0 \leq x \leq 10 D_{\mathrm{j}}$ at $\mathbf{5 0}, \mathbf{1 0 0}$ and $\mathbf{1 5 0}$ time steps (on the fine grid) after the start of the comparison. Red is the refined computation and black is the base computation.

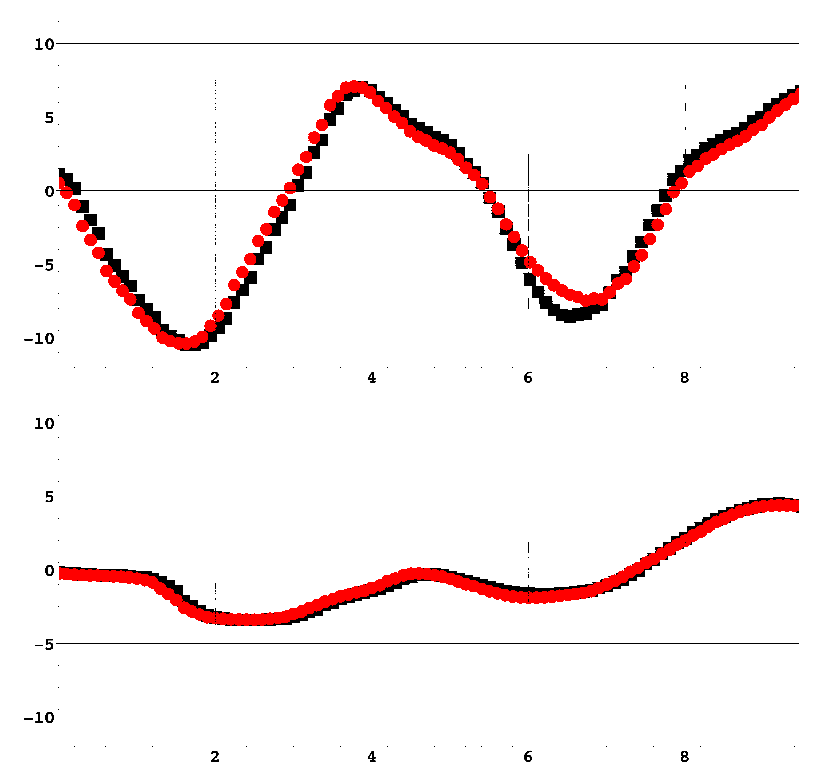

Figure 9. Line plot of the scaled pressure perturbations along the lines between the points $\left(0,0, D_{\mathrm{j}}\right),\left(10 D_{\mathrm{j}}, 0,3 D_{\mathrm{j}}\right)$ (top) and $\left(0,0,4 D_{\mathrm{j}}\right),\left(10 D_{\mathrm{j}}, 0,4 D_{\mathrm{j}}\right)$ (bottom). Red is the refined computation and black is the base computation. The $y$-axis displays $(p-1 / 1.4) / 1.4 \times 1000$ and the $x$ axis displayed the distance from the start point in jet diameters. 